\title{
Improved Mass Accuracy for Tandem Mass Spectrometry
}

\author{
Nathan K. Kaiser \\ Department of Chemistry, Washington State University, Pullman, Washington, USA
}

\section{Gordon A. Anderson}

Biological Sciences Division and Environmental Molecular Sciences Laboratory, Pacific Northwest National Laboratory, Richland, Washington, USA

James E. Bruce

Department of Chemistry, Washington State University, Pullman, Washington, USA

With the emergence of top-down proteomics, the ability to achieve high mass measurement accuracy on tandem MS/MS data will be beneficial for protein identification and characterization. (FT-ICR) Fourier transform ion cyclotron resonance mass spectrometers are the ideal instruments to perform these experiments with their ability to provide high resolution and mass accuracy. A major limitation to mass measurement accuracy in FT-ICR instruments arises from the occurrence of space charge effects. These space charge effects shift the cyclotron frequency of the ions, which compromises the mass measurement accuracy. While several methods have been developed that correct these space charge effects, they have limitations when applied to MS/MS experiments. It has already been shown that additional information inherent in electrospray spectra can be used for improved mass measurement accuracy with the use of a computer algorithm called DeCAL (deconvolution of Coulombic affected linearity). This paper highlights a new application of the strategy for improved mass accuracy in tandem mass analysis. The results show a significant improvement in mass measurement accuracy on complex electron capture dissociation spectra of proteins. We also demonstrate how the improvement in mass accuracy can increase the confidence in protein identification from the fragment masses of proteins acquired in MS/MS experiments. (J Am Soc Mass Spectrom 2005, 16, 463-470) (C) 2004 American Society for Mass Spectrometry

$\mathrm{F}$ ourier Transform Ion Cyclotron Resonance Mass Spectrometry (FT-ICR MS) [1] is a unique technique with its ability to provide simultaneous high resolution, sensitivity, and accurate mass measurements. However, the mass accuracy of FT-ICR is known to be compromised by the influence of space charge effects [2-4], which arise from the Coulombic interaction of trapped ions in the ICR cell. The measured quantity in FT-ICR MS is ion cyclotron frequency. Space charge effects resultant from ion-ion repulsion can produce a systematic shift in the observed cyclotron frequencies. If unaccounted for, these shifts in cyclotron frequency severely degrade mass measurement accuracy. Ion cyclotron frequency and $\mathrm{m} / \mathrm{z}$ are related by eq 1 , and perturbations in the observed cyclotron frequency will correspond to a shift in the $\mathrm{m} / \mathrm{z}$ value. The relationship between $\mathrm{m} / \mathrm{z}$, charge state, and frequency is

Published online February 8, 2005

Address reprint requests to Dr. J. E. Bruce, Department of Chemistry, Washington State University, P.O. Box 644630, Pullman, WA 99164-4630, USA. E-mail: james_bruce@wsu.edu

$$
(m / z)_{n}=\frac{M+n\left(M_{c}\right)}{n}=\frac{k B}{f_{n}}
$$

where $(m / z)_{n}$ is the observed mass-to-charge ratio, $n$ is the number of charges, $M$ is the molecular weight of the analyte being measured, $M_{c}$ is the mass of the charge carrier, $k$ is a proportionality constant relating $\mathrm{m} / \mathrm{z}$ to the magnetic field $B$, and $f_{n}$ is the cyclotron frequency. The magnitude of the frequency shift depends on the changes in the total ion population in the ICR cell during experimental conditions, as compared to the total ion population present during calibration [4]. The frequency shift is constant to a first order approximation across the entire spectrum, in that all ions are shifted by the same amount in frequency space. With a larger number of charge states with increased intensity, possible higher-order non-linear frequency shifts can occur. The systematic shift in ion cyclotron frequency can be expressed by eq 2 where $f$ is the expected cyclotron frequency and $\Delta f$ is the frequency shift that is resultant from the imposed space charge effects. 


$$
m / z=\frac{k B}{(f+\Delta f)}
$$

A number of different calibration functions that convert the ion cyclotron frequency to $m / z$ have been developed $[3,5]$.

Space charge effects limit the mass accuracy and have spurred an active area of research to correct for and reduce this phenomenon. There have been a few methods developed to correct for space charge effects to obtain more accurate mass measurements, while others have reduced the presence of space charge effects by controlling the number of ions that are trapped in the ICR cell [6, 7]. Burton et al. [8] developed a method based on the addition of an internal standard after the initial calibration. Since the frequency shift is constant across the entire spectrum, the added standard will encounter the same shift in frequency space as all the other trapped ions. The frequency shift of the standard can be determined by the difference in observed and expected $\mathrm{m} / \mathrm{z}$ of the added standard. This frequency shift is then applied to all ions present in the ICR cell to obtain improved mass accuracy. Another method developed by Easterling et al. [4] shows that space charge effects can be corrected with a calibration expression that relates the trapped ion population in the ICR cell during calibration to the trapped ion population during the experiment, through a shift in frequency. A third method called Deconvolution of Coulombic Affect Linearity (DeCAL) developed by Bruce et al. [9] corrects for space charge effects in spectra that contain multiply charged ions. This method relies on a computer algorithm that aligns the deconvoluted isotopic distributions of multiple charge states of the same molecular species. Improved mass accuracy was demonstrated by iteratively shifting the entire frequency-domain spectra prior to deconvolution. This is done without knowing any other parameters, such as exact molecular weight, ion abundances, or the identity of the species.

Mass accuracy is a critical feature in the emerging field of proteomics. Proteomics can be defined as the effort to establish identity, structure, and function of all proteins present in the organism, and how these can change in time, space, and other conditions. A newer approach to proteome analysis with mass spectrometry called "topdown proteomics" [10-12], aims to combine protein identification with protein characterization, and locate post-translational modifications (PTMs) present on the proteins. PTMs are important to biological systems because they can change the structure and regulate the functionality of proteins. To determine sequence and PTM sites, proteins have traditionally been proteolytically digested prior to mass spectral analysis. However, some of these PTMs are labile and dissociate easily from the parent ion when performing tandem MS/MS techniques such as CAD (collisionally activated dissociation) or IRMPD (infrared multiphoton dissociation). These methods can potentially lose information on some PTM sites. A unique new dissociation method pioneered by McLafferty et al. called Electron Capture Dissociation (ECD) $[13,14]$ gives a larger number of fragments and sequence coverage than the previously mentioned fragmentation techniques, while preserving labile PTMs intact on the fragment ions [15-18]. This technique offers the capability to locate PTMs from the intact protein without first having to digest the protein. Currently ECD is only compatible with the FTICR mass spectrometer [19]. Although Electron Transfer Dissociation (ETD) reported recently by Hunt et al. [20], enables similar fragmentation pathways to be observed with other types of mass spectrometers, FT-ICR instrumentation uniquely allows simultaneous high resolution, mass accuracy, and sensitivity for complex spectral interpretation. The advantage that high mass accuracy offers to proteomics is its ability to identify and unambiguously confirm protein identification through the production of sequence tag information, and peptide or fragment masses when dealing with the top-down approach.

When analyzing proteins through the bottom up approach it is possible to identify a protein based only on a few peptide masses, when these masses are searched against a database. The number of peptide masses required for positive identification of a protein is decreased as the mass accuracy increases [21-23]. It has been shown that with adequate mass accuracy and additional constraints, it is possible to identify a protein with just one peptide mass [23]. There have been a number of different search tools designed to deal with this type of bottom up analysis of proteins. The same idea applies when dealing with MS/MS fragments of whole proteins instead of peptides; as the mass accuracy of the measurement increases, the number of fragment masses needed to identify a protein is decreased. High mass accuracy will prove to be advantageous when doing chromatographic runs of whole proteins which are heavily modified or when there are multiple proteins present. Since the mass accuracy in ICR technology can be limited by space charge effects, a method to correct for space charge effects in tandem MS/MS experiments to obtain high mass accuracy is needed. Furthermore, the number of ions entering the mass spectrometer during a chromatographic run can vary greatly which will lead to mass errors associated with space charge effects since the number of ions entering the ICR cell is not constant. There have been many research efforts designed to circumvent this problem and obtain high mass accuracy in tandem MS/MS ICR experiments [24-28]. Efforts have also been made to control the number of ions entering the ICR cell; the development of automated gain control limits the need to correct for space charge effects by routinely allowing the same total ion population to enter the ICR cell $[6,7$, 29]. However, during ECD and top-down experiments it is desirable to obtain a large parent ion population in the ICR cell. This is because of the lower efficiency of the dissociation process and the large number of possible fragmentation pathways that result in an increased production of lower intensity fragment ions. If a smaller 
population of precursor ions is used for dissociation, the fragment ions that form to a lesser degree are lost in the noise and not detected. Also, the parent ion population is often not the same as the total ion population after dissociation because of the charge reduction processes that can take place during ECD. Thus, controlling the ion population by limiting the number of ions in the ICR cell can be problematic when doing ECD experiments. Finally, correction of space charge effects with an internal calibrant for MS/MS experiments adds additional complexity to the experiments, such as the need to inject the calibrant species along with the MS/MS fragment ions into the ICR cell. In dissociation spectra such as those resultant from ECD, there are a large number of peaks with a wide range of charge states. This makes the detected time-domain signal more complex due to the amount of constructive and destructive interferences $[30,31]$. The complexity affects the peak intensities in such a way that the summation of the peak intensities may no longer reflect the total ion intensity in the ICR cell [32]. During MS/MS experiments there are a wide range of fragment masses that are formed. The calibration procedure works best when it is possible to accurately determine the exact ion intensity and works best for high mass species when the $\mathrm{m} / \mathrm{z}$ of the calibrant is matched to the analyte [32]. DeCAL only requires that multiple charge states of the same molecular species be present in the spectrum; therefore it will only work with multiply charged ions. During MS/MS experiments there is usually only one charge state that is selected for dissociation. However, for highly charged ions, these species often dissociate in such a way that there are multiple charge states of the same molecular fragment produced. When intact protein ions are analyzed by electrospray and ECD they dissociate to produce many charge state pairs, thus allowing the opportunity for space charge effect correction with DeCAL. The advantage that DeCAL provides is that everything that is needed to correct for space charge effects is inherent in the spectrum. The correction is done through post processing and can be done without any further manipulation of the ion population or previous knowledge of the species being analyzed.

\section{Experimental}

Ubiquitin $\left(\mathrm{MW}_{\mathrm{avg}}=8565 \mathrm{Da}\right)$ and horse myoglobin $\left(\mathrm{MW}_{\mathrm{avg}}=16952 \mathrm{Da}\right)$ were dissolved in a solution of 49:49:2 by volume of water, methanol, and acetic acid and diluted to $10 \mathrm{uM}$. Electrospray was used as the ionization source. The voltage on the capillary was set between $-2050 \mathrm{~V}$ and $-2350 \mathrm{~V}$. A syringe pump used to introduce the solutions was set between $15-25 \mathrm{uL} / \mathrm{h}$. A Bruker Daltonics Apex-Q 7T FT-ICR mass spectrometer (Billerica, MA) was used to acquire the mass spectral data using Xmass as the data acquisition software program. The instrument was externally calibrated using ubiquitin a few days prior to the collection of data presented here. However, careful adjustments of trapped ion population sizes present during data acquisition to match those that were present during calibration was not performed. Therefore, the observed uncorrected error is not what one might expect from a high performance mass analyzer operated under carefully controlled conditions. The ions enter the instrument through a glass capillary and then pass through a hexapole followed by a quadrupole then a second hexapole. The quadrupole was used to select the specific $\mathrm{m} / \mathrm{z}$ ions of interest to be fragmented $(11+$ for ubiquitin, 16+ for myoglobin). These ions were accumulated between 1 to $2 \mathrm{~s}$ in the second hexapole to acquire sufficient ion population for ECD. The ions were then transported to the ICR cell using electrostatic focusing. The ions were trapped in the ICR cell using a low energy sidekick potential to keep the ions close to the central z-axis of the cell. Electron capture dissociation was performed using a heated cathode dispenser located outside the ICR cell to obtain the MS/MS data. The cathode dispenser was heated with 1.6 to $1.8 \mathrm{~A}$ and held at approximately 5 to $6 \mathrm{~V}$. The electron injection time was set at 1.0-3.0 ms, the potential on the solid cathode dispenser was set at -7.5 to $-15 \mathrm{~V}$. The spectra were obtained by signal averaging 65 scans. All data sets acquired were $512 \mathrm{k}$ points. Data were interpreted with aid of the computer program ICR-2LS [33]. The calibration function that was used to convert ion cyclotron frequency to $m / z$ values is written as a variation of eq 2 and represented by eq 3 , where $A$ and $B$ are calibration constants, and $f$ is the measured cyclotron frequency.

$$
m / z=\frac{A}{(f-B)}
$$

The data sets were apodized using the Welch apodization function and zero-filled twice before Fourier transformation to the frequency domain. The DeCAL program used to correct for the frequency shift in the tandem MS/MS data is a module written within ICR2LS. The monoisotopic masses of the fragment ions from the spectra were entered into the web based software and database tool called ProSight PTM, designed by Kelleher et. al. [34] to specifically deal with the characteristics of the top-down proteomics approach. To compare the effects of identification of a protein using fragment masses from ECD data, monoisotopic masses of the fragment ions with and without the use of DeCAL were entered into the ProSight PTM program.

\section{Results and Discussion}

When two charge states of the same molecular species are deconvoluted and overlaid, optimum overlap occurs when the observed frequency shift is equal to the shift resultant from space charge effects. Here our assumption is that the frequency shift is a constant value across the entire spectrum, which seems to be true at least to a first order approximation. However, De- 
No DeCAL

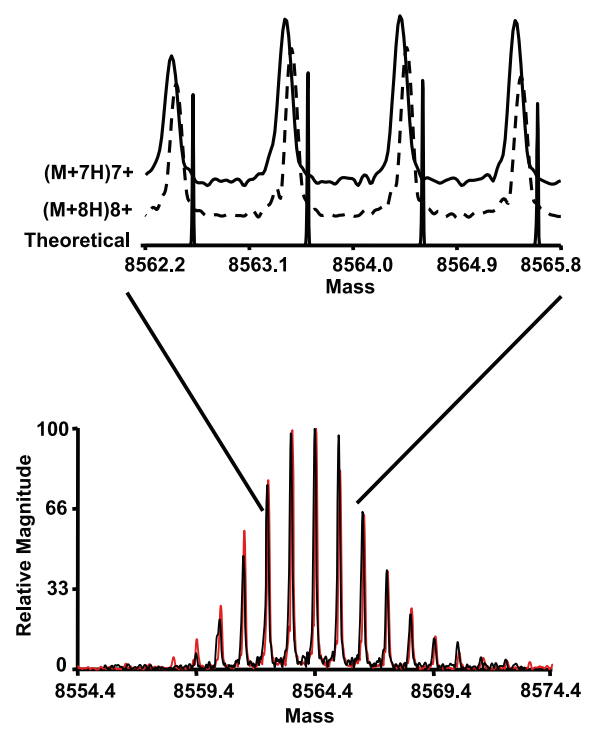

Application of DeCAL

Figure 1. The deconvoluted isotopic distributions of the $8+$ and $7+$ charge states of ubiquitin are overlaid. The spectrum on the left is with no correction of frequency. The expanded region reveals that the two isotopic distributions do not exactly overlap. The theoretical isotopic distribution is also displayed. The spectrum on the right is after the application of DeCAL. Here the isotopic distributions overlap much better, and as the isotopic distributions converge they do so in good agreement with the theoretical isotopic distribution.

CAL or derivatives of this approach can also account for more complex non-linear shifts. This requires that sufficient charge states exist in the spectrum to allow functional characterization of non-linear shifts. A constant shift in frequency space causes different $\mathrm{m} / \mathrm{z}$ values to be shifted to slightly different degrees in the mass domain causing the two deconvoluted isotopic distributions of the same molecular species to not overlap exactly as would be expected. The two isotopic distributions are expected to overlap exactly when deconvoluted because they have the same molecular composition. DeCAL shifts the original frequencydomain spectrum in an iterative fashion in frequencyspace until the resultant two deconvoluted isotopic distributions overlap in the mass-domain. Importantly, when the two isotopic distributions converge, they do so in alignment with the theoretical isotopic distribution as can be seen in Figure 1. In this figure, the theoretical isotopic distribution of ubiquitin is shown, along with deconvolved isotopic distributions resultant from the $8+$ and $7+$ charge states of ubiquitin. Initially, the two isotopic distributions do not overlap extremely well. As the two isotopic distributions are shifted in frequency space they begin to merge together and do so in good agreement with the theoretical isotopic distribution. The error between the deconvoluted isotopic distributions is minimized at the same time that the alignment with the theoretical distribution (which is not normally known, but shown here for illustrative purpose) becomes optimal. When the two deconvoluted isotopic distributions overlap, the shift in frequency is equal to the frequency shift due to the space charge effects. DeCAL acts as a correction process for space charge effects resultant from Coulombic interactions. However, it should be noted that this correction process will be ineffective if the instrument is initially poorly calibrated.

ECD was performed on the $11+$ charge state of ubiquitin (Figure 2), and the resulting fragment ion peaks within $100 \mathrm{ppm}$ error range were identified using ProSight PTM. The average mass error in the spectrum without correction for space charge effects was $53 \mathrm{ppm}$. While better than data from some MS/MS instruments, these results do not match the performance in mass accuracy that is expected from a FT-ICR instrument. It should also be noted that much better performance

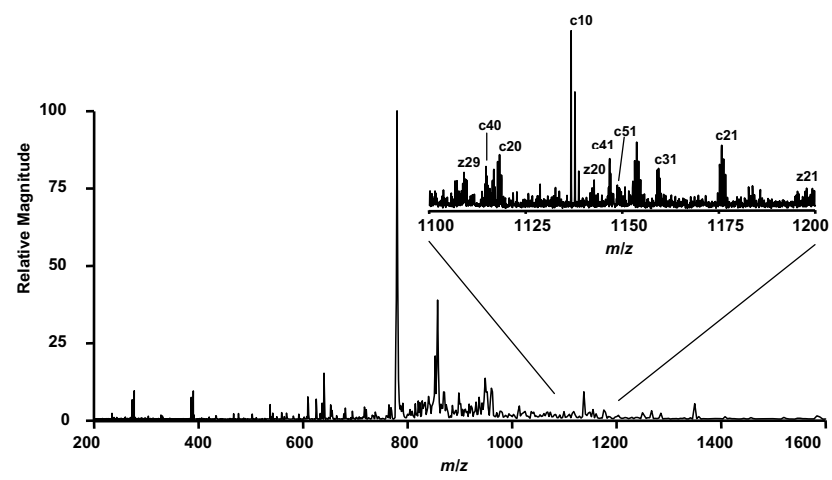

Figure 2. The ECD spectrum of ubiquitin $11+$. The inset shows identification of several $\mathrm{c}$ and $\mathrm{z} \cdot$ ions. 


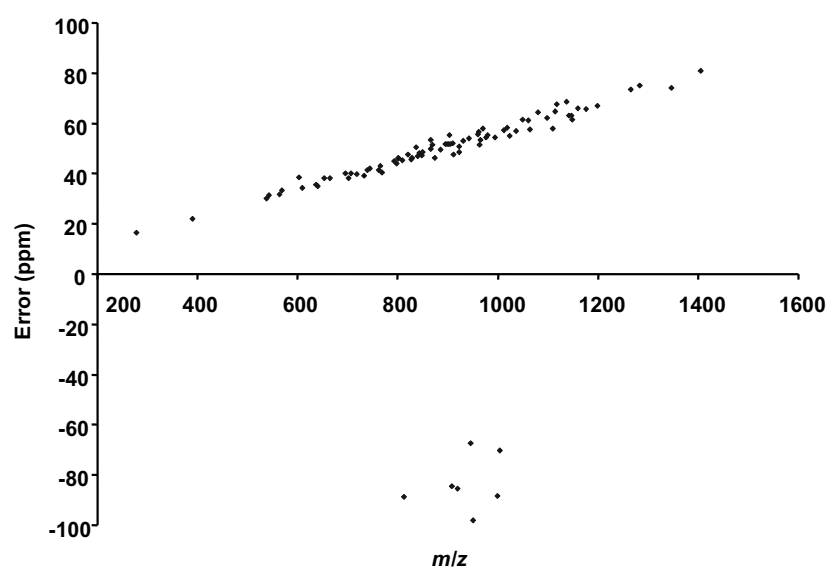

Figure 3. A visual representation of the error associated with the identified isotopic distributions of ubiquitin fragment ions following ECD of the ubiquitin $11+$ ions. The graph shows a systematic increase in error with increasing $\mathrm{m} / \mathrm{z}$. The average error in the spectra was $53.1 \mathrm{ppm}$. Peaks that have misassigned monoisotopic peaks are located in a cluster toward the bottom of the graph.

could have been obtained through careful calibration and matching of total ion populations. This example is used to show the utility of DeCAL to correct space charge effects when the ion populations are not matched. In the absence of sophisticated "automated gain control" type experiments, this mismatch of ion population sizes is very likely in LC/MS or LC/MS/MS experiments. Fortunately, space charge effects result in a systematic error in which a constant offset is applied to all frequencies in the spectrum. Plotting the error in ppm of the identified peaks in the spectrum versus $\mathrm{m} / \mathrm{z}$ as seen in Figure 3, gives a visual indication of the systematic error that occurs. As the $m / z$ value increases, the error also increases in a linear fashion. This increase in error as the $\mathrm{m} / \mathrm{z}$ increases is expected based on the inverse relationship between $\mathrm{m} / \mathrm{z}$ and cyclotron frequency as seen in eq 1 . As the $\mathrm{m} / \mathrm{z}$ value increases, the frequency decreases. Thus, for a constant frequency offset, the higher $\mathrm{m} / \mathrm{z}$ (lower frequency) will exhibit a larger relative shift in frequency and be shifted to a greater extent in the mass-domain than the lower $\mathrm{m} / \mathrm{z}$ (higher frequency) ions. DeCAL was then applied to the same set of acquired data to correct space charge effects. No knowledge of the ion identity or ion abundance was used. A plot of resultant error in ppm of the identified fragment ions versus $m / z$ is shown in Figure 4. This figure shows substantial improvement in error and the feasibility of correction for this type of systematic error in MS/MS spectra. The plotted error points no longer have a linear trend of increasing error, which shows that DeCAL has virtually removed the systematic error. After applying DeCAL to the data, the average error in ppm decreased to less than $2 \mathrm{ppm}$ which is greater than a 30-fold improvement. Thus, DeCAL has the capability of correcting space charge effects in tandem MS/MS experiments used for top-down proteomics.

During the experiment, the $11+$ charge state of

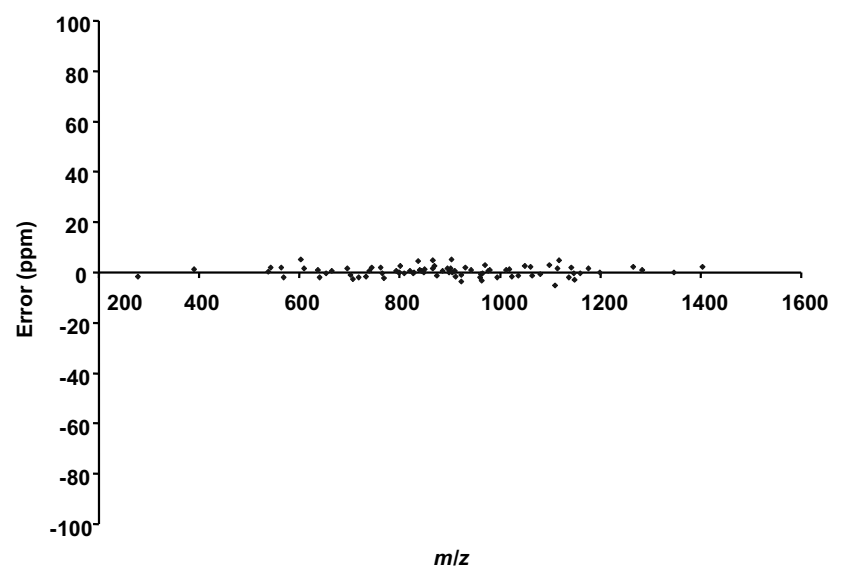

Figure 4. The error associated with the identified isotopic distributions of the fragment ions produced from ECD of the ubiquitin $11+$ ions following the application of DeCAL. The graph shows the correction of the systematic error produced by space charge effects. The average error in the fragment ion spectra after DeCAL application was $1.59 \mathrm{ppm}$. The cluster of misassigned peaks seen in Figure 3 are now shifted out of the 100 ppm search window.

ubiquitin was isolated using the mass selective quadrupole. These ions were then dissociated inside the ICR cell by ECD to give an array of charge states that ranged from $11+$ to $1+$. When the ions dissociated, a large number of isotopic distributions resulted, some of which were the same molecular fragment but with different charge states. This production of charge state pairs is common with dissociation of highly charged precursor ions, and is advantageous since DeCAL relies on charge state pairs to perform its calculations for correction. Within the ubiquitin spectrum, the program was able to locate eleven charge state pairs, and these charge state pairs were used to perform DeCAL. The induced frequency shift for the data set was determined to be $6.03 \mathrm{~Hz}$.

The application of DeCAL to MS/MS data is useful for the top-down proteomic approach. The increased mass accuracy is useful when analyzing fragment masses to determine the identity and possible PTM sites. The data collected from the ECD spectrum of the $16+$ charge state of horse myoglobin (Figure 5) was

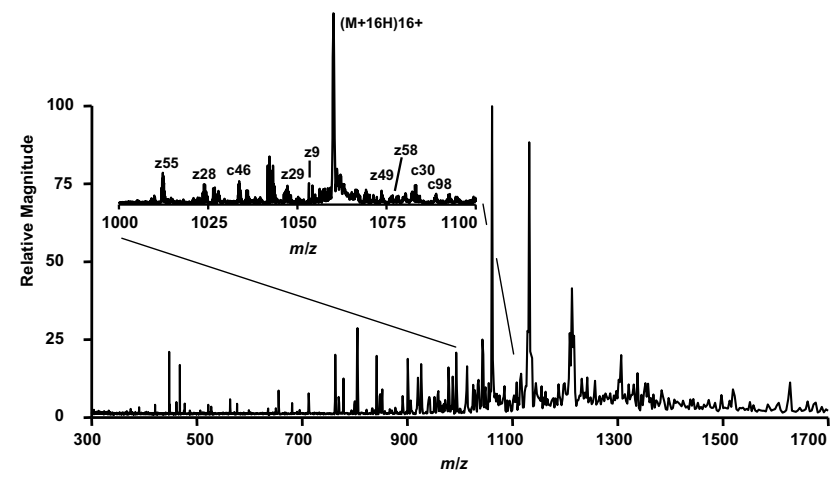

Figure 5. ECD-FTICR mass spectrum of myoglobin $16+$. The inset shows the identification of several $\mathrm{c}$ and $\mathrm{z} \cdot$ ions. 


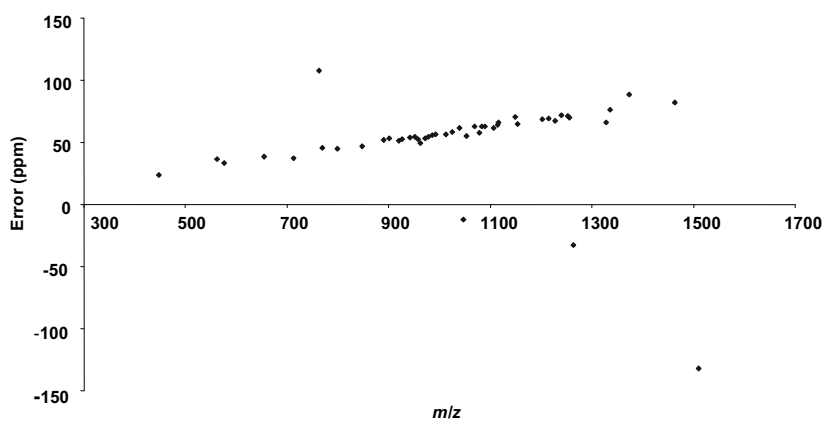

Figure 6. The error associated with the identified isotopic distributions of the fragment ions produced from ECD of the myoglobin $16+$ ion. The average error of the identified peaks in the spectra was $58.9 \mathrm{ppm}$.

used in the database search to determine how the mass accuracy effects the ability to identify the protein based on the fragment masses. ECD produces primarily $c$ and $z$. fragments when the parent ion dissociates, so the search was performed looking for these fragments. Without the correction of space charge effects, 50 monoisotopic masses were selected corresponding to the most intense isotopic distributions in the spectrum, and were entered into ProSight PTM. When setting the parameter for fragment mass tolerance to $25 \mathrm{ppm}$, there were 28 proteins that were identified as possible matches, none of which were myoglobin. After performing DeCAL and running the search at $5 \mathrm{ppm}$ myoglobin is readily identified with a P-score of $8.1 \times$ $10^{-48}$. This P-score gives the probability of a match being a random event, so the lower the P-score the higher the confidence of the search [35]. The average error for the identified fragment ion peaks in the myoglobin raw data was 58.8 ppm. Figure 6 shows this substantial error. The error associated with the spectrum is not too surprising since the FT-ICR instrument was calibrated using ubiquitin, with a different set of parameters and different ion abundances in the ICR cell, a few days prior to obtaining the mass spectrum of myoglobin. Figure 7 shows how the error in ppm

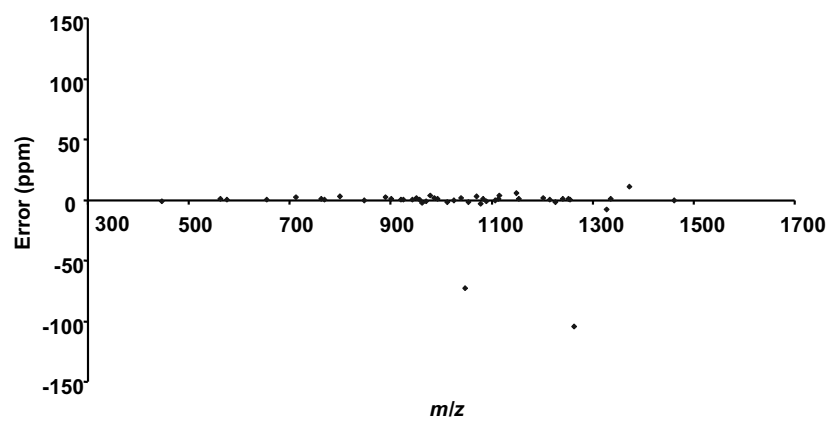

Figure 7. The error associated with the identified isotopic distributions of the fragment ions produced from ECD on the myoglobin $16+$ ion following the application of DeCAL. The application of DeCAL shows a dramatic effect on the distribution and average error in the spectra. The average error in the spectra decreased to $5.5 \mathrm{ppm}$.
Table 1. The top 50 most intense fragment masses from the ubiquitin ECD spectra of the 11+ ions were entered into ProSight PTM. The intact protein mass range was set at 8500 $+/-2000$ Da while the mass tolerance of the fragment masses was varied. The use of DeCAL shows a substantial improvement in the P-Score and in the ability to identify a protein from its fragment masses.

\begin{tabular}{lccccc}
\hline & \multicolumn{2}{c}{ Without DeCAL } & & \multicolumn{2}{c}{ With DeCAL } \\
\cline { 2 - 3 } \cline { 5 - 6 } $\begin{array}{l}\text { Allowed mass } \\
\text { tolerance in } \\
\text { ppm }\end{array}$ & $\begin{array}{c}\text { Fragments } \\
\text { identified }\end{array}$ & P-score & & $\begin{array}{c}\text { Fragments } \\
\text { identified }\end{array}$ & P-score \\
\hline \hline 5 & 0 & - & 22 & $9.84 \mathrm{E}-43$ \\
10 & 0 & - & 22 & $3.80 \mathrm{E}-36$ \\
25 & 0 & - & 22 & $1.71 \mathrm{E}-27$ \\
35 & 3 & 35.92 & 22 & $2.40 \mathrm{E}-24$ \\
50 & 15 & $2.19 \mathrm{E}-11$ & 22 & $4.84 \mathrm{E}-21$ \\
65 & 22 & $1.22 \mathrm{E}-18$ & 22 & $1.22 \mathrm{E}-18$ \\
85 & 23 & $1.90 \mathrm{E}-17$ & 22 & $3.27 \mathrm{E}-16$ \\
100 & 24 & $4.16 \mathrm{E}-17$ & 22 & $9.22 \mathrm{E}-15$ \\
\hline
\end{tabular}

changes after the application of DeCAL on the same set of data. DeCAL indicated a frequency shift of $6.07 \mathrm{~Hz}$ and the average error dropped to $5.5 \mathrm{ppm}$, which is over a factor of 10 improvement. By statistically removing the two outliers, which can be seen in Figure 7, the average error drops to $1.7 \mathrm{ppm}$ which gives about another factor of 3 improvement.

The advantage that DeCAL offers when applied to MS/MS data for this type of analysis is to increase the confidence of obtaining positive protein identification. Ubiquitin was used to test how the probability of a random match is effect by mass accuracy. The top 50 most intense fragment masses from the spectrum were selected and entered into the database. The mass tolerance parameter in ProSight PTM was varied with and without the application of DeCAL (Table 1). Without DeCAL, ubiquitin was not selected as a probable match until the mass tolerance parameter of the search increased to $35 \mathrm{ppm}$. At this tolerance, even though ubiquitin was selected as a probable match, it did not receive the lowest P-score of the proteins selected as a probable match. The probability of a match did not become significant until the mass tolerance was increased to $50 \mathrm{ppm}$, which is also close to the average error of the assigned isotopic peaks (53 ppm) in the data shown in Figure 3. The probability that the identified protein was not a random event reached a maximum at $1.2 \times 10^{-18}$, at which the mass tolerance was set at 65 ppm. With the use of DeCAL, ubiquitin was detected with confidence within a mass tolerance of $5 \mathrm{ppm}$, with the probability of it being a random event being $9.8 \times$ $10^{-43}$. Table 1 shows the impact of database searching with and without the application of DeCAL. In Table 1, the P-scores are lower with the application of DeCAL until the mass tolerance is set to $65 \mathrm{ppm}$, at which the two columns have equal P-scores. The reason is that at 65 ppm the same number of fragment ions have been assigned to ubiquitin for both cases. As the mass tolerance is allowed to increase to $100 \mathrm{ppm}$ the P-score 
without DeCAL becomes less (more significant) than the P-score with DeCAL. The reason for this observation is that there are more peaks assigned to ubiquitin within that mass tolerance. As seen in Figure 3 there is a cluster of identified peaks that fall below the trend line formed due to the systematic error. These peaks are the result of the misassigned monoisotopic peaks in the ECD spectrum. All of the misassigned fragment ions were highly charged and of relatively low abundance so that the monoisotopic peak was not visible in the spectrum. These peaks were actually assigned to the correct fragment. However, since the algorithm misidentified the monoisotopic peaks, the error values from these fragments do not follow the observed trend. When the frequency shift is corrected, these peaks are shifted out of the $100 \mathrm{ppm}$ error window and are not seen in Figure 4. These possible misassigned peaks are taken into account when determining the P-score, which artificially causes the P-score of the uncorrected data to be better than it should be. With the use of DeCAL these possible misassigned peaks are outside of the search tolerance. The application of DeCAL allows the mass tolerance to be minimized, reducing the number of possible misassigned peaks that could possibly lead to incorrect protein identification, thus allowing greater confidence in identification of the intact protein. DeCAL will be even more useful when identifying a protein with unknown modifications, where many of the fragment masses will have modifications associated with them. DeCAL should also prove useful when multiple proteins are present, as might be expected with chromatographic separation of large numbers of proteins. The P-score is based on the mass tolerance window, the number of identified fragments, and the number of masses being searched. Therefore, with multiple proteins present, many of the fragment masses will come from different proteins and the more masses searched that do not fit within the mass tolerance will lead to a higher the P-score. With the application of DeCAL, the mass tolerance range can be decreased which will lead to a decrease in the P-score and a more confident identification can be assigned. Increasing the mass accuracy will also help to find sites of PTM with higher confidence.

\section{Conclusions}

The high mass measurement accuracy in FT-ICR MS can be severely limited by space charge effects. These space charge effects present in tandem MS/MS experiments can be corrected with the application of DeCAL when total ion populations are not well matched with those encountered during calibration. This can be especially problematic in ECD experiments as normally these experiments are carried out with maximum trap capacity of the precursor, and result in variable amounts of product ions. However, ECD spectra often contain a number of charge state pairs that can be used for correction. The application of DeCAL results in an average error for tandem MS/MS data in the low ppm range, even when trapped ion populations are not carefully controlled. This improvement in mass accuracy allows one to accurately identify proteins based on accurate fragment masses. Without the use of DeCAL, our database search did not correctly identify myoglobin protein when the search mass tolerance was set at 25 ppm or better. With DeCAL application, we were able to identify proteins unambiguously with the mass tolerance set to much tighter constraints. These tighter search constraints yield much greater confidence in the search results. Additionally, the higher mass accuracy possible with DeCAL will be beneficial when analyzing complex protein mixtures. Finally, increased mass measurement accuracy will also likely allow more accurate determination of posttranslational sites from top-down proteomic studies.

\section{Acknowledgments}

This research was supported by the National Science Foundation, Instrument Development for Biological Research Program, grant no. DBI-0352451, and the Murdock Charitable Trust. The authors also thank Dr. Gary Kruppa for his assistance with initial ECD experiments.

\section{Appendix Supplementary Data}

Supplementary data associated with this article can be found in the online version, doi:10.1016/j.jasms.2004. 12.005 .

\section{References}

1. Marshall, A. G.; Hendrickson, C. L.; Jackson, G. S. Fourier transform ion cyclotron resonance mass spectrometry: A primer. Mass Spectrom. Rev. 1998, 17(1), 1-35.

2. Ledford, E. B., Jr.; Rempel, D. L.; Gross, M. L. Space charge effects in Fourier transform mass spectrometry. I. Electrons. Int. J. Mass Spectrom. Ion Processes 1984, 55(2), 143-154.

3. Ledford, E. B., Jr.; Rempel, D. L.; Gross, M. L. Space charge effects in Fourier transform mass spectrometry. Mass calibration. Anal. Chem. 1984, 56(14), 2744-2748.

4. Easterling, M. L.; Mize, T. H.; Amster, I. J. Routine part-permillion mass accuracy for high-mass ions: Space-charge effects in MALDI FT-ICR. Anal. Chem. 1999, 71(3), 624-632.

5. Francl, T. J.; Hunter, R. L.; McIver, R. T., Jr. Zoom transform for mass measurement accuracy in Fourier transform mass spectrometry. Anal. Chem. 1983, 55(13), 2094-2096.

6. Belov, M. E.; Zhang, R.; Strittmatter, E. F.; Prior, D. C.; Tang, K.; Smith, R. D. Automated gain control and internal calibration with external ion accumulation capillary liquid chromatography-electrospray ionization Fourier transform ion cyclotron resonance. Anal. Chem. 2003, 75(16), 4195-4205.

7. Belov, M. E.; Rakov, V. S.; Nikolaev, E. N.; Goshe, M. B.; Anderson, G. A.; Smith, R. D. Initial implementation of external accumulation liquid chromatography/electrospray ionization Fourier transform ion cyclotron resonance with automated gain control. Rapid Commun. Mass Spectrom. 2003, 17(7), 627-636.

8. Burton, R. D.; Matuszak, K. P.; Watson, C. H.; Eyler, J. R. Exact mass measurements using a 7 tesla Fourier transform ion 
cyclotron resonance mass spectrometer in a good laboratory practices-regulated environment. J. Am. Soc. Mass Spectrom. 1999, 10(12), 1291-1297.

9. Bruce, J. E.; Anderson, M. D.; Brands, G. A.; Pasa-Tolic, L.; Smith, R. D. Obtaining more accurate Fourier transform ion cyclotron resonance mass measurements without internal standards using multiply charged ions. J. Am. Soc. Mass Spectrom. 2000, 11(5), 416-421.

10. Ge, Y.; Lawhorn, B. G.; ElNaggar, M.; Strauss, E.; Park, J. H.; Begley, T. P.; McLafferty, F. W. Top down characterization of larger proteins $(45 \mathrm{kDa})$ by electron capture dissociation mass spectrometry. J. Am. Chem. Soc. 2002, 124(4), 672-678.

11. Reid, G. E.; McLuckey, S. A. “Top down” protein characterization via tandem mass spectrometry. J. Mass Spectrom. 2002, 37(7), 663-675.

12. Sze, S. K.; Ge, Y.; Oh, H; McLafferty, F. W. Top-down mass spectrometry of a 29-kDa protein for characterization of any posttranslational modification to within one residue. Proc. Natl. Acad. Sci. U.S.A. 2002, 99(4), 1774-1779.

13. Zubarev, R. A.; Kelleher, N. L.; McLafferty, F. W. Electron capture dissociation of multiply charged protein cations. A nonergodic process. J. Am. Chem. Soc. 1998, 120(13), 3265-3266.

14. McLafferty, F. W.; Horn, D. M.; Breuker, K.; Ge, Y.; Lewis, M. A.; Cerda, B.; Zubarev, R. A.; Carpenter, B. K. Electron capture dissociation of gaseous multiply charged ions by Fourier-transform ion cyclotron resonance. J. Am. Soc. Mass Spectrom. 2001, 12(3), 245-249.

15. Kelleher, N. L.; Zubarev, R. A.; Bush, K.; Furie, B.; Furie, B. C.; McLafferty, F. W.; Walsh, C. T. Localization of labile posttranslational modifications by electron capture dissociation: The case of g-carboxyglutamic acid. Anal. Chem. 1999, 71(19), $4250-4253$.

16. Hakansson, K.; Cooper, H. J.; Emmett, M. R.; Costello, C. E.; Marshall, A. G.; Nilsson, C. L. Electron capture dissociation and infrared multiphoton dissociation MS/MS of an N-glycosylated tryptic peptide to yield complementary sequence information. Anal. Chem. 2001, 73(18), 4530-4536.

17. Mirgorodskaya, E.; Roepstorff, P.; Zubarev, R. A. Localization of O-glycosylation sites in peptides by electron capture dissociation in a Fourier transform mass spectrometer. Anal. Chem. 1999, 71(20), 4431-4436.

18. Shi, S. D. H.; Hemling, M. E.; Carr, S. A.; Horn, D. M.; Lindh, I.; McLafferty, F. W. Phosphopeptide/phosphoprotein mapping by electron capture dissociation mass spectrometry. Anal. Chem. 2001, 73(1), 19-22.

19. Zubarev, R. A. Reactions of polypeptide ions with electrons in the gas phase. Mass Spectrom. Rev. 2003, 22(1), 57-77.

20. Syka, J. E.; Coon, J. J.; Schroeder, M. J.; Shabanowitz, J.; Hunt, D. F. Peptide and protein sequence analysis by electron transfer dissociation mass spectrometry. Proc. Natl. Acad. Sci. U.S.A. 2004, 101(26), 9528-9533.

21. Takach, E. J.; Hines, W. M.; Patterson, D. H.; Juhasz, P.; Falick, A. M.; Vestal, M. L.; Martin, S. A. Accurate mass measurements using MALDI-TOF with delayed extraction. J. Prot. Chem. 1997, 16(5), 363-369.

22. Clauser, K. R.; Baker, P.; Burlingame, A. L. Role of accurate mass measurement $( \pm 10 \mathrm{ppm})$ in protein identification strat- egies employing MS or MS/MS and database searching. Anal. Chem. 1999, 71(14), 2871-2882.

23. Goodlett, D. R.; Bruce, J. E.; Anderson, G. A.; Rist, B.; PasaTolic, L.; Fiehn, O.; Smith, R. D.; Aebersold, R. Protein identification with a single accurate mass of a cysteine-containing peptide and constrained database searching. Anal. Chem. 2000, 72(6), 1112-1118.

24. Masselon, C.; Anderson, G. A.; Harkewicz, R.; Bruce, J. E.; Pasa-Tolic, L.; Smith, R. D. Accurate mass multiplexed tandem mass spectrometry for high-throughput polypeptide identification from mixtures. Anal. Chem. 2000, 72(8), 19181924.

25. Kruppa, G.; Schnier, P. D.; Tabei, K.; Van Orden, S.; Siegel, M. M. Multiple ion isolation applications in FT-ICR MS: Exact-mass MSn internal calibration and purification/interrogation of protein-drug complexes. Anal. Chem. 2002, 74(15), 3877-3886.

26. Hannis, J. C.; Muddiman, D.C. A dual electrospray ionization source combined with hexapole accumulation to achieve high mass accuracy of biopolymers in Fourier transform ion cyclotron resonance mass spectrometry. J. Am. Soc. Mass Spectrom. 2000, 11(10), 876-883.

27. Brock, A.; Horn, D. M.; Peters, E. C.; Shaw, C. M.; Ericson, C.; Phung, Q. T.; Salomon, A. R. An automated matrix-assisted laser desorption/ionization quadrupole Fourier transform ion cyclotron resonance mass spectrometer for "bottom-up" proteomics. Anal. Chem. 2003, 75(14), 3419-3428.

28. Chan, T. W. D.; Duan, L.; Sze, T. P. E. Accurate mass measurements for peptide and protein mixtures by using matrix-assisted laser desorption/ionization Fourier transform mass spectrometry. Anal. Chem. 2002, 74(20), 5282-5289.

29. Senko, M.; Zabrouskov, V.; Lange, O.; Wieghaus, A.; Horning, $\mathrm{S}$. LC/MS with external calibration mass accuracies approaching $100 \mathrm{ppb}$. Proceedings of the 52nd ASMS Conference on Mass Spectrometry and Allied Topics; Nashville, TN, May, 2004.

30. Easterling, M. L.; Amster, I. J.; van Rooij, G. J.; Heeren, R. M. A. Isotope beating effects in the analysis of polymer distributions by Fourier transform mass spectrometry. J. Am. Soc. Mass Spectrom. 1999, 10(11), 1074-1082.

31. Hofstadler, S. A.; Bruce, J. E.; Rockwood, A. L.; Anderson, G. A.; Winger, B. E.; Smith, R. D. Isotopic beat patterns in Fourier transform ion cyclotron resonance mass spectrometry: Implications for high resolution mass measurements of large biopolymers. Int. J. Mass Spectrom. Ion Processes 1994, 132(1/2), 109-127.

32. Taylor, P. K.; Amster, I. J. Space charge effects on mass accuracy for multiply charged ions in ESI-FTICR. Int. J. Mass Spectrom. 2003, 222(1/3), 351-361.

33. Anderson, G. A.; Bruce, J. E.; Smith, R. D. ICR-2LS. 1996: Pacific Northwest National Laboratory: Richland, WA.

34. Taylor, G. K.; Kim, Y.-B.; Forbes, A. J.; Meng, F.; McCarthy, R.; Kelleher, N. L. Web and database software for identification of intact proteins using "top down" mass spectrometry. Anal. Chem. 2003, 75(16), 4081-4086.

35. Meng, F.; Cargile, B. J.; Miller, L. M.; Forbes, A. J.; Johnson, J. R; Kelleher, N. L. Informatics and multiplexing of intact protein identification in bacteria and the archaea. Nat. Biotechnol. 2001, 19(10), 952-957. 